\title{
Novel Method of Forecasting Performance of Full Vehicle Suspension by Decoupling Analysis and Vibration Sensing Experiments
}

\author{
Long Wu, ${ }^{1}$ Lei Zuo, ${ }^{2}$ and Kun-Chieh Wang ${ }^{1 *}$ \\ ${ }^{1}$ School of Mechanical and Electronic Engineering, Sanming University, \\ Sanming, 365004 Fujian Province, China \\ ${ }^{2}$ Department of Mechanical Engineering, Virginia Polytechnic Institute and State University, \\ Blacksburg, 24060 Virginia State, USA
}

(Received September 17, 2019; accepted April 2, 2020)

Keywords: full vehicle suspension, performance forecasting, decoupling analysis, independent quarter car models, vibration sensing experiment

A quarter, half, or full car model is often adopted as the basis of vibration control for the design and evaluation of automotive suspension systems. Regarding automotive suspension systems, a quarter vehicle design-and-test result cannot be used as a substitute for a full vehicle owing to the coupling phenomenon. In this study, by deriving the coupling ratios between the sprung mass of a full car and four sprung masses of quarter cars, the analysis of a full vehicle dynamics is performed with 14 degrees of freedom in the conventional vertical and assisted lateral directions. It is found that for car suspensions, the full car model can be conditionally expressed by four independent quarter car models. Moreover, we verify our proposed novel performance forecasting method for a full vehicle suspension through analyses and investigations via suitable designated vibration sensing experiments. Furthermore, a case study shows that the vibration of a full vehicle can be quantitatively obtained on the basis of test results of quarter suspensions.

\section{Introduction}

A good car suspension can provide high ride comfort and driving stability. A quarter vehicle model is often utilized to test and verify complex control strategies and new actuators owing to its simple structure and convenience in experimental environments. Soliman et al ${ }^{(1)}$ showed the effectiveness of the linear matrix inequality (LMI) optimization method in application to car active suspensions. Marek et al. ${ }^{(2)}$ presented a synthesis of a weighted multitone optimal controller and investigated the effect of identification time selection on the vibration transmission function of an active vibration reduction system. Pepe and Carcaterra ${ }^{(3)}$ proposed a variational feedback controller (VFC) theory and applied it to improve a nonlinear quarter suspension performance. Ahmed et al. ${ }^{(4)}$ designed a sophisticated proportional integral and derivative (PID) control for a quarter car model for the automotive industry and validated

*Corresponding author: e-mail: m18316252102@126.com

https://doi.org/10.18494/SAM.2020.2682 
its performance by comparing passive and active suspension systems. The half car model is often used for motorcycle design and the performance validation of the front and rear wheel suspensions for cars. Choi et al. ${ }^{(5)}$ introduced a new dynamic output-feedback controller based on the function-based integral inequality method and the reciprocally convex approach. Ozgur et al. ${ }^{(6)}$ compared classical PID control, fuzzy logic control (FLC), and hybrid fuzzy-PID control (HFPID) based on a half car model. Li et al. ${ }^{(7)}$ constructed a robust controller via convex optimization in terms of feasible LMIs for a half vehicle to show its effectiveness. Zhang et al. ${ }^{(8)}$ realized the independent control of the pitch and vertical motions of a half car using a dual-damper-based controllable quarter car suspension structure. The full car model is accurate for simulating the vibration absorbing capacity of a car owing to its advantages of more degrees of freedom (DOFs) and fitting to an actual vehicle. Aldair and Wang ${ }^{(9)}$ used an artificial intelligent neuro-fuzzy (NF) technique to design a robust controller to improve the dynamic response based on a full vehicle nonlinear active suspension system. Wang et al. ${ }^{(10)}$ proposed a robust dynamic output-feedback controller with the fault tolerance (FT) ability to improve full car performance. Chen et al. ${ }^{(11)}$ presented an efficient $\mathrm{H}_{2}$ optimization method for passive mechanical control problems with a special class of positive real controllers based on a full car model. Du et al. ${ }^{(12)}$ adopted a static output feedback controller to improve vehicle ride comfort in terms of driver head acceleration under constraints of actuator saturation, suspension deflection limitation, and road holding capability. A traditional full car model can be used to study the vibration of a car suspension under the influence of vertical excitation from the road. However, owing to a complicated coupling relationship, the results of a quarter suspension model cannot be quantitatively expanded to a full model even though some researchers have attempted to do so. ${ }^{(13,14)}$ Similar investigation results may also be found in many papers. ${ }^{(15-18)}$

With the development of vehicle dynamics, many researchers seek a composite model to solve vibration problems for nonlinear motions. In past studies, the lateral excitation between tires and ground in steering was considered to study three car body motions based on the conventional vertical model. ${ }^{(13,15)}$ However, the adoption of lateral excitation requires more DOFs in a vehicle model. For example, considering vertical and lateral excitations simultaneously, a full vehicle model should include the vertical and lateral motions of four unsprung masses; the vertical, lateral, pitch, roll, and yaw motions of the sprung mass; and the vertical vibration of the driver. This results in 14 DOFs. Although more DOFs could describe the dynamics more accurately, it also leads to greater computational load and control complexity than the conventional car model only with vertical analysis. Therefore, to simplify the model, new methods should be developed.

In automobile theory, there is a concept called the mass distribution coefficient (MDC) for a half car model. When the distances from the front and rear wheels to the center of the sprung mass are the same, the MDC equals one. The whole sprung mass can be separated into two sprung masses belonging to the front and rear quarter car models. The front sprung mass has the same magnitude as the rear sprung mass. Thus, a half car model can be translated into two independent quarter car models. From this, we conclude that the sprung mass should have coupling actions with all wheel systems. However, the coupling action existing in a full car model has rarely been introduced. Therefore, if the research on the MDC is extended to a full 
car, the coupling relationships between four-wheel systems and the full car can be identified clearly.

Owing to the near symmetry of a four-wheel car, a full car can be seen as a combination of four quarter vehicles. On this basis, the coupling relationships between a full vehicle and quarter vehicles can be determined. In recent years, a hierarchical modeling method has been developed to construct a parallel control framework with four relative independent quarter suspensions to realize this idea. ${ }^{(16)}$ However, a means of obtaining full car data based on the experimental results of vibration sensing experiment results for a quarter car and considering only effects in the vertical direction has not been pursued, although such an approach would be attractive. In this study, with the assistance of the lateral factor based on the conventional vertical vehicle model, five motions of the car body are examined by space force analysis to deduce the vertical coupling ratios between the continuous sprung mass of a full car and four independent sprung masses of quarter cars. In this way, an approach to quantitatively acquire the focused vibration finding of a full car by using the experimental results of quarter suspensions is established. Therefore, a novel performance analysis method for a full vehicle suspension is presented for use in the vehicle design and test fields. Furthermore, various smart sensors and microprocessors can be employed in vehicle suspension systems, including those of a full, half, or quarter vehicle. ${ }^{(17,18)}$ By performing some suitable vibration sensing experiments, we verify the proposed performance forecasting model. In detail, the system parameters of a full or quarter vehicle system can be fully understood using system identification technologies. ${ }^{(19-22)}$ Thus, the proposed performance forecasting model can be set up via sensors with an appropriate experimental arrangement.

\section{Decoupling Analysis}

A car body only has vertical, pitch, and roll motions in traditional research based on vertical excitations under wheels. However, many cars have four-wheel systems. Thus, the dynamical relationships between the three motions of the car body and four supporting forces provided by four-wheel systems can be found if we separately consider a car body. Conventionally, in the analysis of a car vibration control, the car is always assumed to move in a uniform, straight trajectory. Thus, the lateral excitations generated by the effect of tires can be ignored. In addition, if we consider the lateral and yaw motions of a car body, the relationship between car body motions and supporting forces may be obtained through the introduction of lateral excitations.

Figure 1 depicts the free-body diagram of a car sprung mass with suspension forces. The suspension spring, damping, and controllable forces at each corner of the sprung mass are combined to model a concentrated vertical force $F_{i z}(i=1-4)$. Each tire also exerts a lateral force $F_{i y}(i=1-4)$ at each corner of the sprung mass. The spring and damping forces of the driver system are also simplified to a concentrated force $F_{p}$.

The dynamic equations of the five-DOF sprung mass can be obtained in terms of the vertical displacement $z_{c}$, lateral displacement $y_{c}$, pitch $\theta_{c}$, roll $\phi_{c}$, and yaw $\varphi_{c}$ of the mass center. 


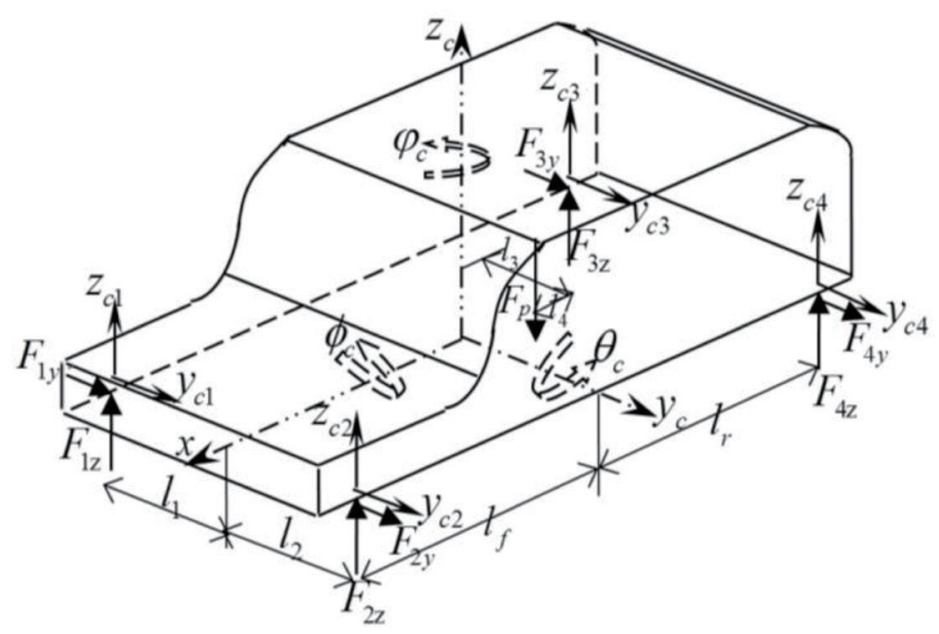

Fig. 1. Force analysis in space for a vehicle suspension sprung mass.

$$
\begin{gathered}
m_{c} \ddot{z}_{c}=F_{1 z}+F_{2 z}+F_{3 z}+F_{4 z}-F_{p} \\
m_{c} \ddot{y}_{c}=F_{1 y}+F_{2 y}+F_{3 y}+F_{4 y} \\
I_{y} \ddot{\theta}_{c}=-l_{f} F_{1 z}-l_{f} F_{2 z}+l_{r} F_{3 z}+l_{r} F_{4 z}+l_{4} F_{p} \\
I_{x} \ddot{\phi}_{c}=l_{1} F_{1 z}-l_{2} F_{2 z}+l_{1} F_{3 z}-l_{2} F_{4 z}+l_{3} F_{p} \\
I_{z} \ddot{\varphi}_{c}=l_{f} F_{1 y}+l_{f} F_{2 y}-l_{r} F_{3 y}-l_{r} F_{4 y}
\end{gathered}
$$

If the forces $F_{i z}$ and $F_{i y}$ act as two component forces, the resultant force $F_{i}$ can be substituted with the two component forces in the above equations. The resulting expressions of the resultant forces are given in Appendix.

The relationships between the vertical and lateral displacements of the center of the sprung mass and each corner can be obtained from the following geometric relationships:

$$
\begin{aligned}
& z_{c 1}=z_{c}-l_{f} \theta_{c}+l_{1} \phi_{c}, \\
& z_{c 2}=z_{c}-l_{f} \theta_{c}-l_{2} \phi_{c}, \\
& z_{c 3}=z_{c}+l_{r} \theta_{c}+l_{1} \phi_{c}, \\
& z_{c 4}=z_{c}+l_{r} \theta_{c}-l_{2} \phi_{c}, \\
& z_{c p}=-z_{c}+l_{4} \theta_{c}+l_{3} \phi_{c}, \\
& y_{c 1}=y_{c}+l_{f} \varphi_{c}+l_{1} \phi_{c},
\end{aligned}
$$




$$
\begin{aligned}
& y_{c 2}=y_{c}+l_{f} \varphi_{c}-l_{2} \phi_{c}, \\
& y_{c 3}=y_{c}-l_{r} \varphi_{c}+l_{1} \phi_{c}, \\
& y_{c 4}=y_{c}-l_{r} \varphi_{c}-l_{2} \phi_{c} .
\end{aligned}
$$

Substituting Eq. (6) into Eq. (1) and multiplying its result by $l_{r}$, then subtracting the final result from Eq. (3), we obtain

$$
m_{c} l_{r} \ddot{z}_{c 1}+m_{c} l_{f} l_{r} \ddot{\theta}_{c}-I_{y} \ddot{\theta}_{c}-m_{c} l_{r} l_{1} \ddot{\phi}_{c}=\left(l_{f}+l_{r}\right) F_{1 z}+\left(l_{f}+l_{r}\right) F_{2 z}-\left(l_{r}+l_{4}\right) F_{p} .
$$

Adding the product of the above equation and $l_{2}$ to the product of Eq. (4) and $\left(l_{f}+l_{r}\right)$ gives

$$
\begin{gathered}
m_{c} l_{r} l_{2} \ddot{z}_{c 1}+m_{c} l_{f} l_{r} l_{2} \ddot{\theta}_{c}-I_{y} l_{2} \ddot{\theta}_{c}-m_{c} l_{r} l_{1} l_{2} \ddot{\phi}_{c}+I_{x}\left(l_{f}+l_{r}\right) \ddot{\phi}_{c}= \\
\left(l_{f}+l_{r}\right)\left(l_{1}+l_{2}\right) F_{1 z}+\left(l_{f}+l_{r}\right) l_{1} F_{3 z}-\left(l_{f}+l_{r}\right) l_{2} F_{4 z}-\left(l_{r}+l_{4}\right) l_{2} F_{p}+\left(l_{f}+l_{r}\right) l_{3} F_{p} .
\end{gathered}
$$

Similarly,

$$
\begin{gathered}
m_{c} l_{r} l_{1} \ddot{z}_{c 2}+m_{c} l_{f} l_{r} l_{1} \ddot{\theta}_{c}-I_{y} l_{1} \ddot{\theta}_{c}+m_{c} l_{r} l_{1} l_{2} \ddot{\phi}_{c}-I_{x}\left(l_{f}+l_{r}\right) \ddot{\phi}_{c}= \\
\left(l_{f}+l_{r}\right)\left(l_{1}+l_{2}\right) F_{2 z}-\left(l_{f}+l_{r}\right) l_{1} F_{3 z}+\left(l_{f}+l_{r}\right) l_{2} F_{4 z}-\left(l_{r}+l_{4}\right) l_{1} F_{p}-\left(l_{f}+l_{r}\right) l_{3} F_{p}, \\
m_{c} l_{f} l_{2} \ddot{z}_{c 3}-m_{c} l_{f} l_{r} l_{2} \ddot{\theta}_{c}+I_{y} l_{2} \ddot{\theta}_{c}-m_{c} l_{f} l_{1} l_{2} \ddot{\phi}_{c}+I_{x}\left(l_{f}+l_{r}\right) \ddot{\phi}_{c}= \\
\left(l_{f}+l_{r}\right) l_{1} F_{1 z}-\left(l_{f}+l_{r}\right) l_{2} F_{2 z}+\left(l_{f}+l_{r}\right)\left(l_{1}+l_{2}\right) F_{3 z}-\left(l_{f}-l_{4}\right) l_{2} F_{p}+\left(l_{f}+l_{r}\right) l_{3} F_{p}, \\
m_{c} l_{f} l_{1} \ddot{z}_{c 4}-m_{c} l_{f} l_{r} l_{1} \ddot{\theta}_{c}+I_{y} l_{1} \ddot{\theta}_{c}+m_{c} l_{f} l_{1} l_{2} \ddot{\phi}_{c}-I_{x}\left(l_{f}+l_{r}\right) \ddot{\phi}_{c}= \\
-\left(l_{f}+l_{r}\right) l_{1} F_{1 z}+\left(l_{f}+l_{r}\right) l_{2} F_{2 z}+\left(l_{f}+l_{r}\right)\left(l_{1}+l_{2}\right) F_{4 z}-\left(l_{f}-l_{4}\right) l_{1} F_{p}-\left(l_{f}+l_{r}\right) l_{3} F_{p} .
\end{gathered}
$$

Adding the above four equations results in

$$
m_{c 1} \ddot{z}_{c 1}+m_{c 2} \ddot{z}_{c 2}+m_{c 3} \ddot{z}_{c 3}+m_{c 4} \ddot{z}_{c 4}=F_{1 z}+F_{2 z}+F_{3 z}+F_{4 z}-F_{p} .
$$

The sum of Eqs. (15) and Eq. (18) is multiplied by $l_{r}$. The difference between this quantity and the sum of Eqs. (16) and (17) multiplied by $l_{f}$ is

$$
I_{y}^{h} \ddot{\theta}_{c}=-l_{f} F_{1 z}^{h}-l_{f} F_{2 z}^{h}+l_{r} F_{3 z}^{h}+l_{r} F_{4 z}^{h}+l_{4} F_{p} .
$$

Using a similar process, the sum of Eqs. (16) and (18) is multiplied by $l_{1}$, and the difference 
between this and the sum of Eqs. (17) and (19) multiplied by $l_{2}$ is

$$
I_{x}^{h} \ddot{\phi}_{c}=l_{1} F_{1 z}^{h h}-l_{2} F_{2 z}^{h h}+l_{1} F_{3 z}^{h h}-l_{2} F_{4 z}^{h h}+l_{3} F_{p} .
$$

Next, substituting Eq. (11) into Eq. (2) and substituting the results in Eqs. (3)-(5) give

$$
\begin{gathered}
m_{c 1} \ddot{y}_{c 1}+m_{c 2} \ddot{y}_{c 2}+m_{c 3} \ddot{y}_{c 3}+m_{c 4} \ddot{y}_{c 4}=F_{1 y}+F_{2 y}+F_{3 y}+F_{4 y}, \\
I_{z}^{h} \ddot{\varphi}_{c}=l_{f} F_{1 y}^{h}+l_{f} F_{2 y}^{h}-l_{r} F_{3 y}^{h}-l_{r} F_{4 y}^{h},
\end{gathered}
$$

where $F_{i z}^{h}=F_{i z}-m_{c i} \ddot{z}_{c i}, F_{i z}^{h h}=F_{i z}-m_{c i} \ddot{z}_{c i} / 2$, and $F_{i y}^{h}=F_{i y}-m_{c i} \ddot{y}_{c i}$. After decoupling, the pitch, roll, and yaw inertia of the full vehicle are $I_{y}^{h}=I_{y}-m_{c} l_{f} l_{r}, I_{x}^{h}=I_{x}-m_{c} l_{1} l_{2} / 2$, and $I_{z}^{h}=I_{z}-m_{c} l_{f} l_{r}$, respectively. The equivalent masses at each corner are

$$
\begin{aligned}
& m_{c 1}=\frac{l_{r} l_{2}}{\left(l_{f}+l_{r}\right)\left(l_{1}+l_{2}\right)} m_{c}, \\
& m_{c 2}=\frac{l_{r} l_{1}}{\left(l_{f}+l_{r}\right)\left(l_{1}+l_{2}\right)} m_{c}, \\
& m_{c 3}=\frac{l_{f} l_{2}}{\left(l_{f}+l_{r}\right)\left(l_{1}+l_{2}\right)} m_{c}, \\
& m_{c 4}=\frac{l_{f} l_{1}}{\left(l_{f}+l_{r}\right)\left(l_{1}+l_{2}\right)} m_{c} .
\end{aligned}
$$

The derived results indicate that Eqs. (20)-(24) have a similar form to Eqs. (1)-(5), respectively. However, the original sprung mass of a full suspension has been substituted by four sprung masses of quarter suspensions. Moreover, if $l_{f}=l_{r}$ and $l_{1}=l_{2}$, the mathematical expressions of the decoupling sprung masses show that $m_{c 1}, m_{c 2}, m_{c 3}$, and $m_{c 4}$ are each a quarter of $m_{c}$. If $l_{f} \neq l_{r}$ and $l_{1} \neq l_{2}$, the coupling ratio relationship between a whole car and four quarter cars can still be determined. Thus, the MDC of a full vehicle under vertical and lateral road excitations has been derived. It is clear that a full vehicle can be seen as a combination of four quarter vehicles. The five motions of the center of the full suspension sprung mass center are equivalent to the four vertical and lateral motions of the quarter suspension sprung masses. Therefore, if the connective coupling actions vanish, a full sprung mass can be decomposed into four sprung masses of quarter suspensions.

Owing to the effectiveness of a continuous sprung mass, each quarter vehicle sprung mass is subjected to internal coupling actions. If each quarter vehicle sprung mass is independent and lacks the adjacent limitations of other sprung masses, the vertical and lateral positions 
of each decoupled quarter sprung mass will change. If the vertical and lateral displacement variables of each sprung mass supported by each wheel system are respectively assigned as the variables $\Delta z_{c i}$ and $\Delta y_{c i}$ in the process of removing adjacent constraints, the vertical and lateral displacements of each decomposed sprung mass are as follows.

$$
\begin{gathered}
z_{1}=z_{c 1}+\Delta z_{c 1} \\
z_{2}=z_{c 2}+\Delta z_{c 2} \\
z_{3}=z_{c 3}-\Delta z_{c 3} \\
z_{4}=z_{c 4}-\Delta z_{c 4} \\
y_{1}=y_{c 1}+\Delta y_{c 1} \\
y_{2}=y_{c 2}-\Delta y_{c 2} \\
y_{3}=y_{c 3}+\Delta y_{c 3} \\
\Delta \ddot{y}_{c 3}=\frac{y_{4}=y_{c 4}-\Delta y_{c 4}}{2 m_{c 3}} F_{3}-\frac{1}{2} \ddot{y}_{c}+\frac{l_{r}}{2} \ddot{\varphi}_{c}-\frac{l_{1}}{2} \ddot{\phi}_{c} \\
\Delta \ddot{y}_{c 2}=-\frac{\cos \gamma_{1}}{2 m_{c 1}} F_{1}-\frac{1}{2} \ddot{z}_{c}+\frac{l_{f}}{2} \ddot{\theta}_{c}-\frac{l_{1}}{2} \ddot{\phi}_{c} \\
\Delta \ddot{z}_{c 2}=\frac{\sin \gamma_{2}}{2 m_{c 2}} F_{2}-\frac{1}{2} \ddot{z}_{c 1}+\frac{l_{f}}{2} \ddot{y}_{c}+\frac{l_{2}}{2} \ddot{\phi}_{c} \\
\cos \gamma_{c 4} \ddot{z}_{c}+\frac{l_{f}}{2} \ddot{\varphi}_{c}-\frac{l_{2}}{2} \ddot{\phi}_{c} \\
2 m_{c 3} \ddot{z}_{c}+\frac{l_{f}}{2} \ddot{\theta}_{c}+\frac{1}{2} \ddot{\theta}_{c}+\frac{l_{2}}{2} \ddot{\phi}_{c} \\
\ddot{z}_{c} \ddot{\theta}_{c}+\frac{l_{1}}{2} \ddot{\phi}_{c}
\end{gathered}
$$




$$
\Delta \ddot{y}_{c 4}=-\frac{\sin \gamma_{4}}{2 m_{c 4}} F_{4}+\frac{1}{2} \ddot{y}_{c}-\frac{l_{r}}{2} \ddot{\varphi}_{c}-\frac{l_{2}}{2} \ddot{\phi}_{c}
$$

The above investigation of the vehicle suspension dynamics reveals the coupling ratio relationship between a full vehicle and four quarter vehicles. In the traditional vibration control field, a quarter suspension model with two DOFs is usually set up in a laboratory to estimate the effectiveness of a new control strategy or actuator. However, the test results cannot be used to acquire the detailed vibration behavior of a full vehicle. Here, a simulation approach is employed to quantitatively determine the vibration behavior of a full vehicle based on the test results of a quarter suspension model. Note that because of the unknown forces of each quarter suspension supporting the car body at the start of the computation, a suitable fitting loop process should be constructed. A flow chart of the fitting loop in the simulation is shown in Fig. 2. Firstly, three pre-estimated values of the vertical, pitch angle, and roll angle accelerations should be calculated from the given vertical excitations under the four wheels. Secondly, four pre-estimated vertical accelerations and forces of four corners of the car body are obtained. Then, the decoupled pre-estimated sprung mass accelerations of the four quarter vehicles are determined. Finally, if the difference between the pre-estimated and tested values of the quarter vehicle sprung mass is less than a given minimum value, the calculation can be considered effective and the three motions of the full car body can be obtained.

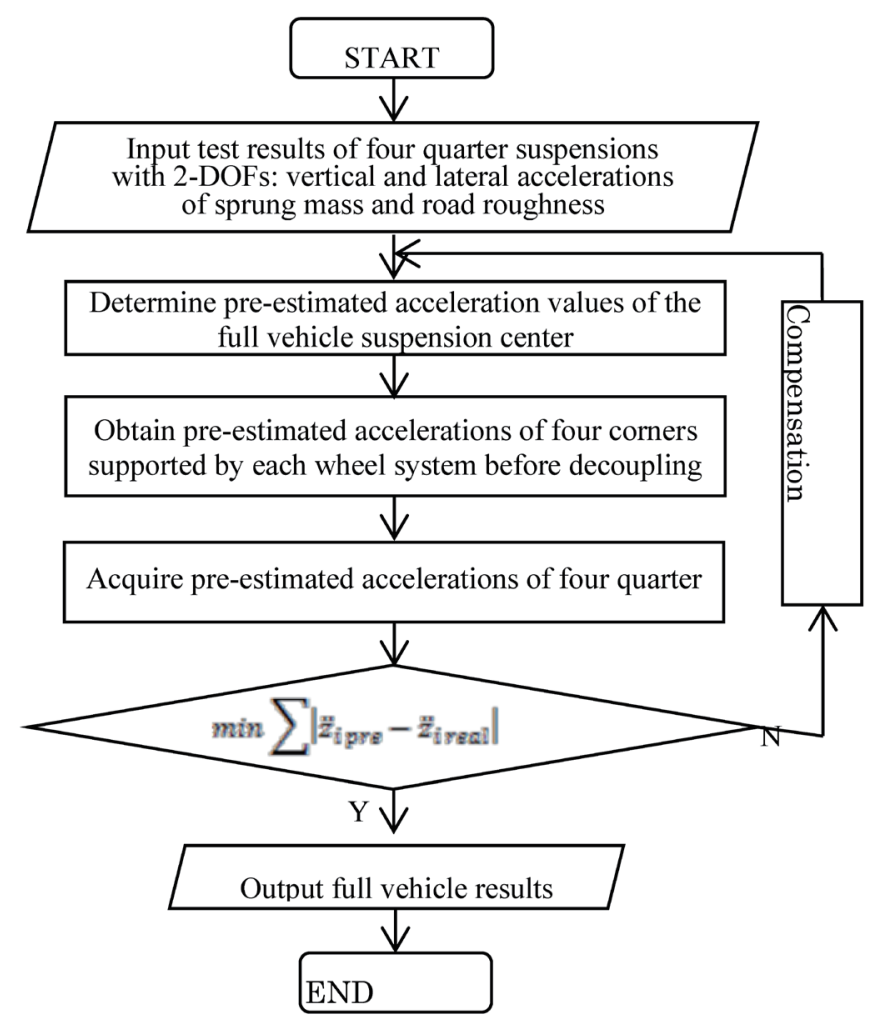

Fig. 2. Flow chart of computation. 
Before attempting to fit the data, the five expected state values of a full vehicle subjected to vertical excitations under the wheels should be determined in advance. This enables a more accurate quarter suspension test. In other words, the quarter suspension test is compatible with the restrictions on the full vehicle structure parameters.

According to the vertical and lateral road roughnesses of each quarter suspension, five pre-estimated variables of the full vehicle sprung mass center containing vertical, lateral, pitch angle, roll angle, and yaw angle accelerations are determined as follows.

$$
\begin{gathered}
\ddot{y}_{c}=\sigma_{1}\left(y_{e 1}+y_{e 2}+y_{e 3}+y_{e 4}\right) /\left(4 t_{s}^{2}\right) \\
\ddot{y}_{c}=\sigma_{2}\left(y_{e 1}+y_{e 2}+y_{e 3}+y_{e 4}\right) /\left(4 t_{s}^{2}\right) \\
\ddot{\theta}_{c}=\sigma_{3}\left\{\operatorname{tg}^{-1}\left[\left(z_{e 3}+z_{e 4}\right)-\left(z_{e 1}+z_{e 2}\right)\right] /\left[2\left(l_{f}+l_{r}\right)\right]\right\} / t_{s}^{2} \\
\ddot{\phi}_{c}=\sigma_{4}\left\{\operatorname{tg}^{-1}\left[l_{f}\left(z_{e 4}-z_{e 3}\right)+l_{r}\left(z_{e 2}-z_{e 1}\right)\right] /\left[\left(l_{f}+l_{r}\right)\left(l_{1}+l_{2}\right)\right]\right\} / t_{s}^{2} \\
\ddot{\varphi}_{c}=\sigma_{5}\left\{\operatorname{tg}^{-1}\left[\left(y_{e 2}-y_{e 1}\right)-\left(y_{e 4}-y_{e 3}\right)\right] /\left(l_{f}+l_{r}\right)\right\} / t_{s}^{2}
\end{gathered}
$$

The coefficient $\sigma_{j}(j=1-5)$ is initially set to 1 , and then adjusted automatically to match the above Eqs. (45)-(49) when the full suspension is in the passive mode. Otherwise, it should be given a small value if the full suspension can be controlled.

Because most dampers and springs are vertically mounted, the vertical impacts of the road can be reduced. In this situation, the vertical, pitch angle, and roll angle accelerations of the full vehicle are all important parameters. Owing to the small values of the lateral excitations generated by tires and the uncontrolled road status, the lateral and yaw angle accelerations of the full vehicle will remain but to a lesser extent. Thus, the lateral excitation should only be utilized to obtain the vertical characteristics of the full suspension and should not be considered for a car moving in a uniform, straight trajectory.

\section{Results and Discussion}

In Sect. 2, a novel performance analysis method for a full vehicle suspension for use in the vehicle design and test fields was presented. This method provides a means of quantitatively acquiring the detailed vibration behavior of a full car by using the experimental results of quarter suspensions.

To ensure that the forecasting method achieves the expected objective, the following parameters of a typical car are taken from a relevant reference, Ref. 2: $m_{p}=80 \mathrm{~kg} ; m_{c}=1182 \mathrm{~kg}$; $I_{x}=1020 \mathrm{~kg} \cdot \mathrm{m}^{2} ; I_{y}=2130 \mathrm{~kg} \cdot \mathrm{m}^{2} ; I_{z}=615 \mathrm{~kg} \cdot \mathrm{m}^{2} ; l_{f}=1.1 \mathrm{~m} ; l_{r}=1.5 \mathrm{~m} ; l_{1}=l_{2}=0.8 \mathrm{~m} ; l_{3}=0.45 \mathrm{~m} ;$ $l_{4}=0.25 \mathrm{~m}$. 
To simplify the computation, C- and A-grade road roughnesses are respectively adopted as the vertical and lateral inputs, as shown in Fig. 3. From Eqs. (25) to (28), four quarter suspension sprung masses are acquired. $m_{c 1}$ and $m_{c 2}$ are $250 \mathrm{~kg}$, whereas $m_{c 3}$ and $m_{c 4}$ are $341 \mathrm{~kg}$. The force of each quarter suspension can be controlled from 200 to $2000 \mathrm{~N}$. Four simulated quarter suspension results obtained before and after decoupling are analyzed using Matlab software and shown in Fig. 4. The acceleration results of the front left and front right sprung masses obtained after decoupling are similar to the active control results in Ref. 2. Table 1 shows a numerical comparison of quarter suspensions in this study and Ref. 2 . In suspension No. 1, the differences are $7.4 \%$ for the maximum sprung mass $(\delta)$ and $5.0 \%$ for the standard deviation of the sprung mass $(\sigma)$. In suspension No. 2, the differences are $5.5 \%$ for $\delta$

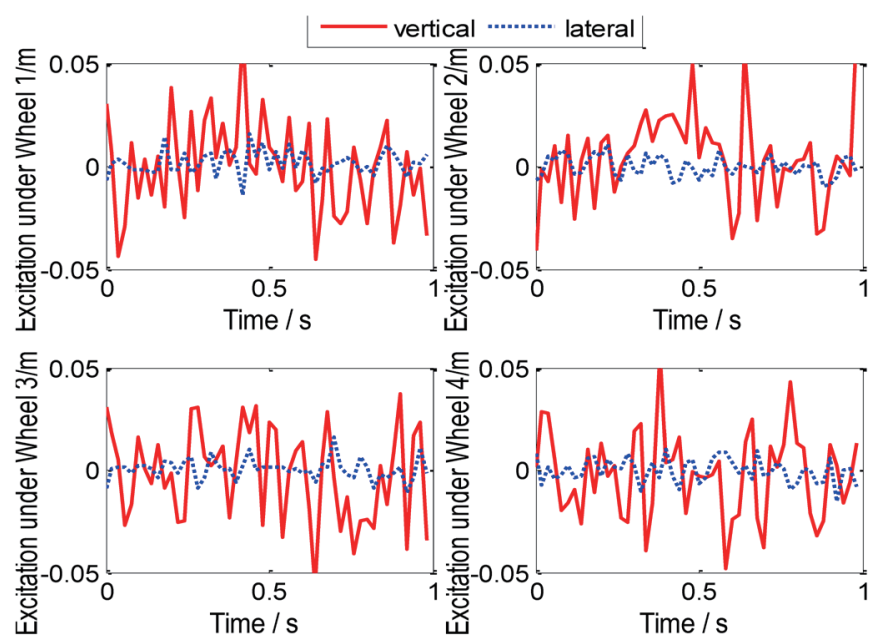

Fig. 3. (Color online) Road excitations under wheels.
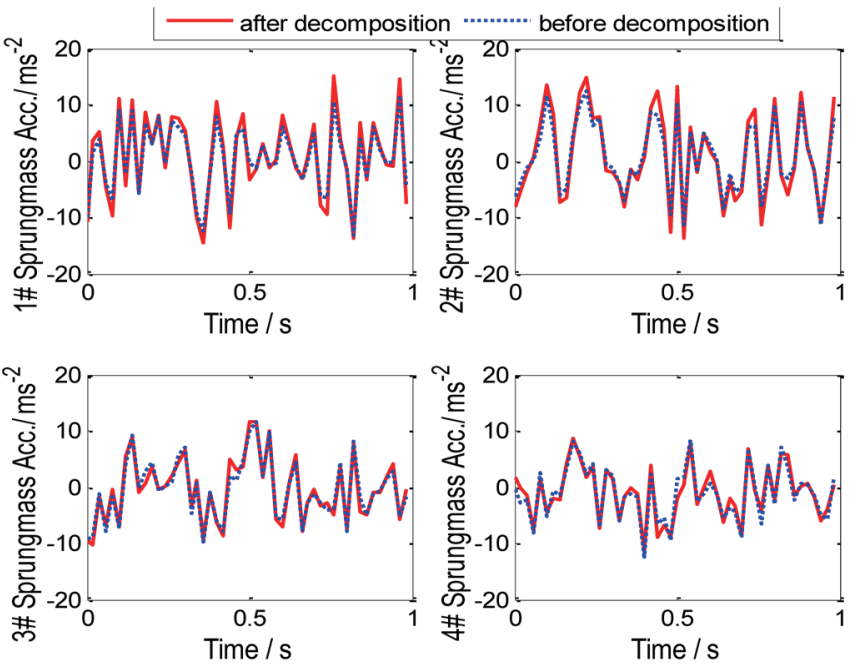

Fig. 4. (Color online) Sprung mass of each wheel. 
Table 1

Numerical comparison of two quarter suspensions.

\begin{tabular}{llrrc}
\hline No. & \multicolumn{1}{c}{ Parameter } & Ref. 2 & This paper & Difference (\%) \\
\hline \multirow{2}{*}{ Suspension No. 1 } & Maximum sprung mass acceleration & 11.68 & 12.54 & 7.4 \\
& Standard deviation of sprung mass acceleration & 6.83 & 7.17 & 5.0 \\
\hline \multirow{2}{*}{ Suspension No. 2 } & Maximum sprung mass acceleration & 11.68 & 12.32 & 5.5 \\
& Standard deviation of sprung mass acceleration & 6.83 & 7.09 & 3.8 \\
\hline
\end{tabular}

Table 2

Comparison of parameters obtained before and after decoupling.

\begin{tabular}{llcrc}
\hline No. & \multicolumn{1}{c}{ Parameter } & After decoupling & Before decoupling & Difference (\%) \\
\hline \multirow{2}{*}{ Suspension No. 1 } & Maximum sprung mass acceleration & 14.44 & 13.15 & 8.9 \\
& Standard deviation of sprung mass acceleration & 7.33 & 5.97 & 18.6 \\
\hline \multirow{2}{*}{ Suspension No. 2} & Maximum sprung mass acceleration & 14.80 & 12.47 & 15.7 \\
& Standard deviation of sprung mass acceleration & 7.78 & 6.33 & 18.6 \\
\hline \multirow{2}{*}{ Suspension No. 3 3} & Maximum sprung mass acceleration & 11.70 & 11.72 & -0.2 \\
& Standard deviation of sprung mass acceleration & 5.64 & 5.46 & 3.2 \\
\hline \multirow{2}{*}{ Suspension No. 4} & Maximum sprung mass acceleration & 11.23 & 12.44 & -10.8 \\
& Standard deviation of sprung mass acceleration & 4.64 & 4.71 & -1.5 \\
\hline
\end{tabular}

and $3.8 \%$ for $\sigma$. It is deduced that the status of suspension No. 2 is better than that of suspension No. 1; thus, suspension No. 2 can be adopted further.

On the basis of the manipulation procedures of the front left and front right quarter suspensions, similar simulation results of two rear quarter suspensions can be obtained using the same conditions. Table 2 shows the parameters of each quarter suspension sprung mass obtained before and after decoupling. Before decoupling, the four quarter suspensions were connected as a full vehicle suspension. After decoupling, the four quarter suspensions could be considered as independent. Using Eqs. (29) to (36), the variation amplitude of each sprung mass of the quarter suspensions, which is given by the standard deviation of the sprung mass acceleration, was obtained. It is clear that the values of the two front quarter suspensions decreased, while those of the two rear quarter suspension values increased. This means that the whole suspension sprung mass was lowered, resulting in an overall downward movement of the front side and an overall upward movement of the rear left corner.

Figures 5 and 6 show the vibration results of the full vehicle suspension sprung mass over time. These figures also show how vertical acceleration values of the full suspension were reduced by the constraint on coupling. The PSD values of the frequency range from 1 to $10 \mathrm{~Hz}$, to which humans are sensitive, decreased and are presented in Table 3. The first natural frequency of the full suspension is preliminarily calculated to be about $1.2 \mathrm{~Hz}$, and those of the independent front and rear quarter suspensions are respectively about 1.3 and $1.1 \mathrm{~Hz}$, as illustrated in Table 4. Obviously, the PSD values of vertical, pitch, and roll motions are much lower at each natural frequency in both the full and quarter suspension models. Even though the vibration of the full suspension can be controlled, the amplitude of vibration in the time domain is still much higher than those in other reports. Therefore, the controllable forces should be enhanced further so as to improve the ride comfort and driving stability. 


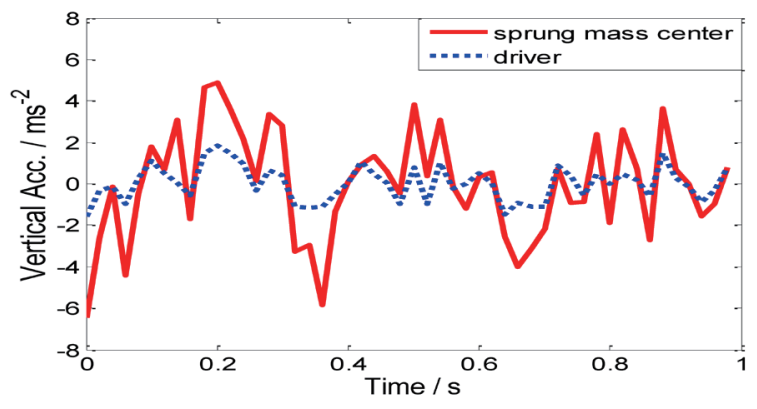

(a)

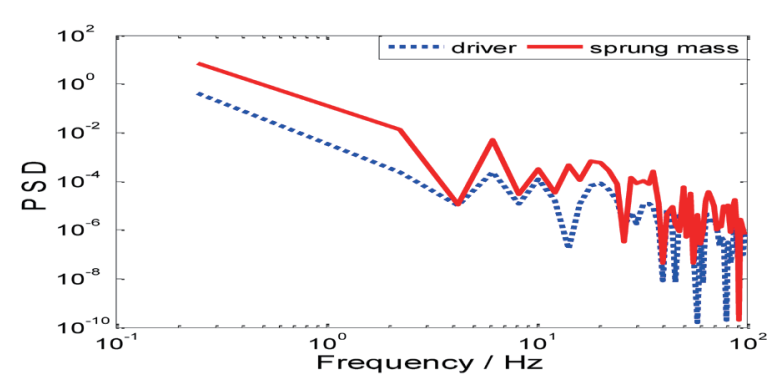

(b)

Fig. 5. (Color online) Vertical vibrations of whole sprung mass and driver. (a) Time domain and (b) frequency domain.

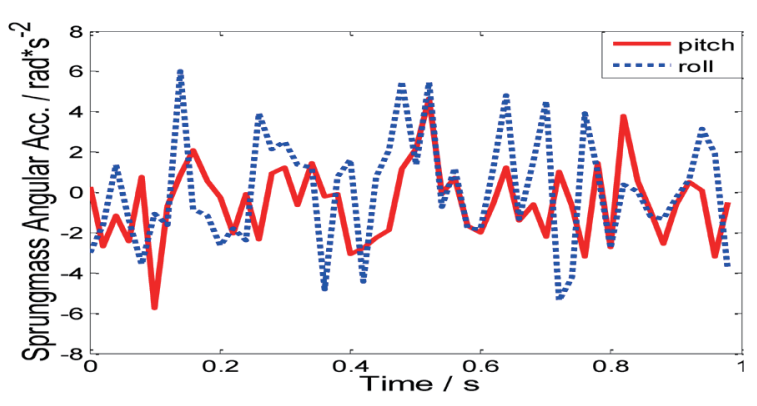

(a)

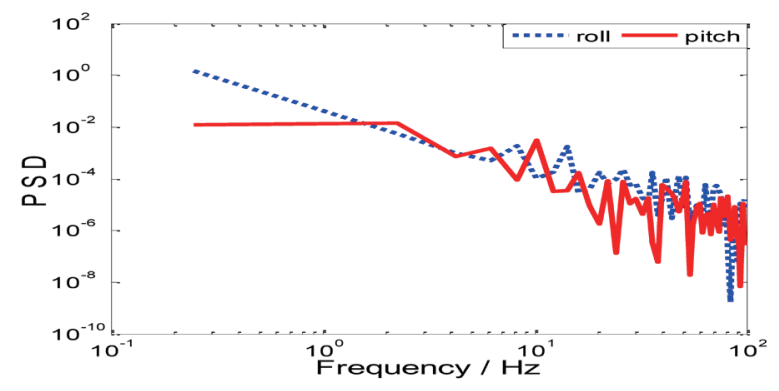

(b)

Fig. 6. (Color online) Pitch and roll angles of whole sprung mass. (a) Time domain and (b) frequency domain.

Table 3

PSD values at different frequencies to which humans are sensitive.

\begin{tabular}{lcccccccccc}
\hline \multirow{2}{*}{ Driver } & $1 \mathrm{~Hz}$ & $2 \mathrm{~Hz}$ & $3 \mathrm{~Hz}$ & $4 \mathrm{~Hz}$ & $5 \mathrm{~Hz}$ & $6 \mathrm{~Hz}$ & $7 \mathrm{~Hz}$ & $8 \mathrm{~Hz}$ & $9 \mathrm{~Hz}$ & $10 \mathrm{~Hz}$ \\
\cline { 2 - 11 } & 0.25 & 0.05 & $1 \mathrm{e}-4$ & $1 \mathrm{e}-4$ & $1 \mathrm{e}-4$ & $2 \mathrm{e}-4$ & $1 \mathrm{e}-4$ & $1 \mathrm{e}-4$ & $5 \mathrm{e}-5$ & $1 \mathrm{e}-4$ \\
\hline
\end{tabular}

Table 4

PSD values for different suspensions at natural frequencies.

\begin{tabular}{lccc}
\hline & $\begin{array}{c}\text { Full suspension } \\
(1.2 \mathrm{~Hz})\end{array}$ & $\begin{array}{c}\text { Front quarter suspension } \\
(1.3 \mathrm{~Hz})\end{array}$ & $\begin{array}{c}\text { Rear quarter suspension } \\
(1.1 \mathrm{~Hz})\end{array}$ \\
\hline Vertical & 0.44 & 0.06 & 0.003 \\
Pitch & 0.01 & - & - \\
Roll & 0.17 & - & - \\
\hline
\end{tabular}

\section{Conclusions}

By considering lateral excitation and force analysis in space, we studied the vibration relationship between a full suspension sprung mass and quarter suspension sprung masses. From the derived formula and case study of simulation, we found that the ratio relationship between a full car suspension and quarter car suspensions can be used to construct a forecasting method. Thus, a relationship connecting a full vehicle and quarter vehicles is established. A 
case study has been carried out and the results are satisfying. It is deduced from the fitting data of a full vehicle suspension that the vibration of a full vehicle can be quantitatively analyzed using the test results of quarter suspensions. Therefore, the forecasting method proposed in this paper provides a new test method for investigating the performance characteristics of a car.

\section{Acknowledgments}

The authors acknowledge the financial support from the Training Program of Outstanding Discipline and Specialty Leaders of Fujian Province Universities and Institutions, the China postdoctoral fund (2013M541851), and the Operational Funding of the Advanced Talents for Scientific Research (19YG04) supported by Sanming University. Technical supports from the following institutions are also acknowledged: Fujian Public Service Platform for miniEREV Power System, Fujian Provincial University Engineering Research Center for Modern Mechanical Design and Manufacturing Technology, Fujian Provincial Collaborative Innovation Center for Green Casting, Forging and Advanced Manufacturing, and Fujian Provincial Engineering Research Center for Casting and Forging Parts.

\section{References}

1 M. Soliman, B. Abdellah, and Y. Hassan: J. Vibr. Control 22 (2016) 258. https://doi. org/10.1177/1077546314528230

2 S. Marek, R. Waldemar, and K. Jarosław: Solid State Phenomena 248 (2016) 127. https://doi.org/10.4028/www. scientific.net/ssp. 248.127

3 G. Pepe and A. Carcaterra: Mech. Syst. Signal Process. 76-77 (2016) 72. https://doi.org/10.1016/ j.ymssp.2016.01.002

4 A. E. S. Ahmed, A. S. Ali, and N. M. Ghazaly Nouby: Int. J. Adv. Eng. Tech. 8 (2015) 899.

5 H. D. Choi, C. K. Ahn, and M. T. Lim, and M. K. Song: Int. J. Control Autom. Syst. 14 (2016) https://doi. org $/ 59.2016 .10 .1007 / \mathrm{s} 12555-015-2005-8$

6 D. Ozgur, K. Ilknur, and C. Saban: Nonlinear Dyn. 67 (2012) 2139. https://doi.org/10.1007/s11071-011-0135-y

7 H. Li, H. Liu, H. Chris, and H. Steve: J. Vibr. Control 19 (2016) 560. https://doi.org/10.1177/1077546311434972

8 H. Zhang, N. Zhang, and F. Min: Math. Prob. Eng. 2016 (2016) 1. https://doi.org/10.1155/2016/1932107

9 A. A. Aldair and W. J. Wang: J. Vibr. Control 18 (2011) 1837. https://doi.org/10.1177/1077546311428631

10 R. Wang, J. Hui, K. H. Reza: Mech. Syst. Signal Process. 62-63 (2015) 341. https://doi.org/10.1016/ j.ymssp.2015.01.015

11 M. Z. Q. Chen, Y. Hu, and F. Wang: J. Dyn. Syst. Meas. Control 137 (2015) 1. https: //doi.org/10.1115/1.4031630

12 H. Du, W. Li, and N. Zhang: Int. J. Veh. Design 63 (2013) 159. https://doi.org/10.1504/ijvd.2013.056133

13 Č. Mirko, J. Deur, X. Li, H. E. Tseng, and D. Hrovat: Veh. Syst. Dyn. 54 (2016) 1004. https://doi.org/10.1080/ 00423114.2016.1177655

14 S. Yu, F. Wang, J. Wang, and H. Chen: Int. J. Veh. Design 68 (2015) 37. https://doi.org/10.4028/www.scientific. net/AMM.456.10

15 J. G. Chen: Appl. Mech. Mater. 456 (2014) 10. https://doi.org/10.4028/www.scientific.net/ AMM.456.10

16 L. Wu: The 2nd Int. Conf. Mechanic Automation and Control Engineering (IEEE) 1 (2011) 147. https://doi. org/10.1109/MACE.2011.5986879

17 H. Gao, W. Sun, and P. Shi: IEEE Trans. Control Syst. Tech. 18 (2010) 238. https://doi.org/10.1109/ TCST.2009.2015653.

18 Y. Guan, Q. L. Han, H. Yao, and X. Ge: Nonlinear Dyn. 94 (2018) 627. https://doi.org/10.1007/s11071-0184381-0

19 Y. Kyongsu and H. Karl: J. Dyn. Syst. Meas. Control 117 (1995). https://doi.org/10.1115/1.2835177.

20 J. Marzbanrad, G. Ahmadi, H. Zohoor, and Y. Hojjat: J. Sound Vibr. 275 (2004) 973. https://doi.org/10.1016/ s0022-460x(03)00812-5.

21 P. Gáspár, Z. Szabo, and J. Bokor: Int. J. Veh. Syst. Model. Testing 2 (2007). https://doi.org/10.1504/ ijvsmt.2007.013891. 
22 I. Youn, M. A. Khan, N. Uddin, E. Youn, and M. Tomizuka: Int. J. Auto. Tech. 18 (2017) 307. https://doi. org/10.1007/s12239.017.0031.7.

\title{
Nomenclature
}

\author{
$m_{p} \quad$ driver system mass \\ $m_{c} \quad$ sprung mass of full vehicle \\ $m_{c i} \quad$ sprung masses of quarter vehicles \\ $I_{y} \quad$ pitch inertia of full vehicle \\ $I_{x} \quad$ roll inertia of full vehicle \\ $I_{z} \quad$ yaw inertia of full vehicle \\ $\theta_{c} \quad$ pitch angle \\ $\phi_{c} \quad$ roll angle \\ $\varphi_{c} \quad$ yaw angle \\ $z_{c} \quad$ vertical displacement of full suspension center \\ $y_{c} \quad$ lateral displacement of full suspension center \\ $z_{c p} \quad$ vertical displacement of sprung mass connected to driver system \\ $z_{c i} \quad$ vertical displacements at four corners of full sprung mass \\ $y_{c i} \quad$ lateral displacements at four corners of full sprung mass \\ $z_{i} \quad$ vertical displacements of independent quarter vehicle sprung masses \\ $\ddot{z}_{i \text { pre }} \quad$ pre-estimated vertical accelerations of quarter suspension sprung masses \\ $\ddot{z}_{i \text { real }} \quad$ actual test accelerations of quarter suspension sprung masses \\ $y_{i} \quad$ lateral displacements of independent quarter vehicle sprung masses \\ $z_{e i} \quad$ vertical excitations under wheels \\ $y_{e i} \quad$ lateral excitations generated by tire effect \\ $F_{i z} \quad$ vertical forces at four corners of full sprung mass \\ $F_{i y} \quad$ lateral forces at four corners of full sprung mass \\ $F_{i} \quad$ resultant force of vertical and lateral forces \\ $F_{p} \quad$ vertical force between full sprung mass and driver systems \\ $\gamma_{i} \quad$ angle between vertical and resultant forces \\ $l_{f} \quad$ front axle distance to full sprung mass center \\ $l_{r} \quad$ rear axle distance to full sprung mass center \\ $l_{1} \quad$ right side distance to full sprung mass center \\ $l_{2} \quad$ left side distance to full sprung mass center \\ $l_{3} \quad$ lateral distance of driver system to full sprung mass center \\ $l_{4} \quad$ longitudinal distance of driver system to full sprung mass center
}




\section{Appendix}

$$
\begin{aligned}
& F_{1}=\lambda_{11} \lambda m_{c} \ddot{z}_{c}+\lambda_{12} \lambda m_{c} \ddot{y}_{c}+\lambda_{13} \lambda I_{y} \ddot{\theta}_{c}+\lambda_{14} \lambda I_{x} \ddot{\phi}_{c}+\lambda_{15} \lambda I_{z} \ddot{\varphi}_{c} \\
& F_{2}=\lambda_{21} \lambda m_{c} \ddot{z}_{c}+\lambda_{22} \lambda m_{c} \ddot{y}_{c}+\lambda_{23} \lambda I_{y} \ddot{\theta}_{c}+\lambda_{24} \lambda I_{x} \ddot{\phi}_{c}+\lambda_{25} \lambda I_{z} \ddot{\varphi}_{c} \\
& F_{3}=\lambda_{31} \lambda m_{c} \ddot{z}_{c}+\lambda_{32} \lambda m_{c} \ddot{y}_{c}+\lambda_{33} \lambda I_{y} \ddot{\theta}_{c}+\lambda_{34} \lambda I_{x} \ddot{\phi}_{c}+\lambda_{35} \lambda I_{z} \ddot{\varphi}_{c} \\
& F_{4}=\lambda_{41} \lambda m_{c} \ddot{z}_{c}+\lambda_{42} \lambda m_{c} \ddot{y}_{c}+\lambda_{43} \lambda I_{y} \ddot{\theta}_{c}+\lambda_{44} \lambda I_{x} \ddot{\phi}_{c}+\lambda_{45} \lambda I_{z} \ddot{\varphi}_{c} \\
& F_{p}=\lambda_{51} \lambda m_{c} \ddot{z}_{c}+\lambda_{52} \lambda m_{c} \ddot{y}_{c}+\lambda_{53} \lambda I_{y} \ddot{\theta}_{c}+\lambda_{54} \lambda I_{x} \ddot{\phi}_{c}+\lambda_{55} \lambda I_{z} \ddot{\varphi}_{c} \\
& \lambda=\sin \gamma_{1} \sin \gamma_{3} \cos \gamma_{2} \cos \gamma_{4} l\left(l_{3}-l_{2}\right)+\sin \gamma_{1} \sin \gamma_{4} \cos \gamma_{2} \cos \gamma_{3}\left(l_{2}\left(l_{r}+l_{4}\right)+l_{1}\left(l_{4}-l_{f}\right)-l l_{3}\right)+ \\
& \sin \gamma_{2} \sin \gamma_{3} \cos \gamma_{1} \cos \gamma_{4}\left(l_{2}\left(l_{f}-l_{4}\right)-l_{1}\left(l_{r}+l_{4}\right)-l l_{3}\right)+\sin \gamma_{2} \sin \gamma_{4} \cos \gamma_{1} \cos \gamma_{3} l\left(l_{1}+l_{3}\right) \\
& \lambda_{11}=\left[\sin \gamma_{3} \cos \gamma_{4}\left(l_{r} l_{3}+l_{2} l_{4}\right)+\sin \gamma_{4} \cos \gamma_{3}\left(l_{1} l_{4}-l_{r} l_{3}\right)\right] \sin \gamma_{2} \\
& \lambda_{12}=\sin \gamma_{3} \cos \gamma_{2} \cos \gamma_{4} l_{r}\left(l_{2}-l_{3}\right)+\sin \gamma_{4} \cos \gamma_{2} \cos \gamma_{3}\left[l l_{3}-l_{2}\left(l_{r}+l_{4}\right)+l_{1}\left(l_{f}-l_{4}\right)\right] l_{r} / l- \\
& \sin \gamma_{2} \cos \gamma_{3} \cos \gamma_{4} l_{f}\left(l_{1}+l_{2}\right)\left(l_{r}+l_{4}\right) / l \\
& \lambda_{13}=\sin \gamma_{2} \sin \gamma_{3} \cos \gamma_{4}\left(l_{2}-l_{3}\right)+\sin \gamma_{2} \sin \gamma_{4} \cos \gamma_{3}\left(l_{1}+l_{3}\right) \\
& \lambda_{14}=\left(\sin \gamma_{3} \cos \gamma_{4}-\sin \gamma_{4} \cos \gamma_{3}\right) \sin \gamma_{2}\left(l_{r}+l_{4}\right) \\
& \lambda_{15}=\sin \gamma_{2} \cos \gamma_{3} \cos \gamma_{4}\left(l_{1}+l_{2}\right)\left(l_{r}+l_{4}\right) / l+\sin \gamma_{3} \cos \gamma_{2} \cos \gamma_{4}\left(l_{2}-l_{3}\right)+ \\
& \sin \gamma_{4} \cos \gamma_{2} \cos \gamma_{3}\left(l_{1}\left(l_{f}-l_{4}\right)-l_{2}\left(l_{r}+l_{4}\right) / l+l_{3}\right) \\
& \lambda_{22}=\sin \gamma_{3} \cos \gamma_{1} \cos \gamma_{4}\left(l_{2}\left(l_{f}-l_{4}\right)-l l_{3}-l_{1}\left(l_{r}+l_{4}\right)\right) l_{r} / l+\sin \gamma_{4} \cos \gamma_{1} \cos \gamma_{3} l_{r}\left(l_{1}+l_{3}\right)- \\
& \sin \gamma_{1} \cos \gamma_{3} \cos \gamma_{4}\left(l_{1}+l_{2}\right)\left(l_{r}+l_{4}\right) l_{f} / l \\
& \lambda_{23}=\left[\sin \gamma_{3} \cos \gamma_{4}\left(l_{2}-l_{3}\right)+\sin \gamma_{4} \cos \gamma_{3}\left(l_{1}+l_{3}\right)\right] \sin \gamma_{1} \\
& \lambda_{24}=\left(\sin \gamma_{3} \cos \gamma_{4}-\sin \gamma_{4} \cos \gamma_{3}\right) \sin \gamma_{1}\left(l_{r}+l_{4}\right) \\
& \lambda_{25}=\sin \gamma_{1} \cos \gamma_{3} \cos \gamma_{4}\left(l_{1}+l_{2}\right)\left(l_{r}+l_{4}\right) / l+\sin \gamma_{4} \cos \gamma_{1} \cos \gamma_{3}\left(l_{1}+l_{3}\right)+ \\
& \sin \gamma_{3} \cos \gamma_{1} \cos \gamma_{4}\left[l_{2}\left(l_{f}-l_{4}\right)-l_{1}\left(l_{r}+l_{4}\right) / l-l_{3}\right] \\
& \lambda_{31}=\sin \gamma_{1} \sin \gamma_{4} \cos \gamma_{2}\left(l_{2} l_{4}-l_{f} l_{3}\right)+\sin \gamma_{2} \sin \gamma_{4} \cos \gamma_{1}\left(l_{f} l_{3}+l_{1} l_{4}\right) \\
& \lambda_{32}=\sin \gamma_{1} \cos \gamma_{2} \cos \gamma_{4} l_{f}\left(l_{3}-l_{2}\right)+\sin \gamma_{2} \cos \gamma_{1} \cos \gamma_{4}\left(l_{2}\left(l_{f}-l_{4}\right)-l_{1}\left(l_{r}+l_{4}\right)-l l_{3}\right) l_{f} / l+ \\
& \sin \gamma_{4} \cos \gamma_{1} \cos \gamma_{2}\left(l_{f}-l_{4}\right)\left(l_{1}+l_{2}\right) / l \\
& \lambda_{33}=\sin \gamma_{1} \sin \gamma_{4} \cos \gamma_{2}\left(l_{2}-l_{3}\right)+\sin \gamma_{2} \sin \gamma_{4} \cos \gamma_{1}\left(l_{1}+l_{3}\right) \\
& \lambda_{34}=\left(\sin \gamma_{2} \cos \gamma_{1}-\sin \gamma_{1} \cos \gamma_{2}\right) \sin \gamma_{4}\left(l_{f}-l_{4}\right) \\
& \lambda_{35}=\sin \gamma_{1} \cos \gamma_{2} \cos \gamma_{4}\left(l_{2}-l_{3}\right)+\sin \gamma_{2} \cos \gamma_{1} \cos \gamma_{4}\left[l_{3}+l_{2}\left(l_{4}-l_{f}\right)+l_{1}\left(l_{r}+l_{4}\right) / l\right]+ \\
& \sin \gamma_{4} \cos \gamma_{1} \cos \gamma_{2}\left[\left(l_{1}+l_{2}\right)\left(l_{f}-l_{4}\right)\right] / l
\end{aligned}
$$




$$
\begin{aligned}
& \lambda_{41}=\sin \left(\gamma_{e 1}\right) \sin \left(\gamma_{e 3}\right) \cos \left(\gamma_{e 2}\right) l_{f} l_{3}-\sin \left(\gamma_{e 2}\right) \sin \left(\gamma_{e 3}\right) \cos \left(\gamma_{e 1}\right) l_{1} l_{4}- \\
& \sin \left(\gamma_{e 2}\right) \sin \left(\gamma_{e 3}\right) \cos \left(\gamma_{e 1}\right) l_{f} l_{3}-\sin \left(\gamma_{e 1}\right) \sin \left(\gamma_{e 3}\right) \cos \left(\gamma_{e 2}\right) l_{2} l_{4} \\
& \lambda_{42}=\sin \gamma_{1} \cos \gamma_{2} \cos \gamma_{3}\left\{\left[l_{f} l_{2}\left(l_{r}+l_{4}\right)+l_{f} l_{1}\left(l_{4}-l_{f}\right)\right] / l-l_{f} l_{3}\right\}+ \\
& \sin \gamma_{2} \cos \gamma_{1} \cos \gamma_{3} l_{f}\left(l_{1}+l_{3}\right)+\sin \gamma_{3} \cos \gamma_{1} \cos \gamma_{2}\left(l_{4}-l_{f}\right)\left(l_{1}+l_{2}\right) l_{r} / l \\
& \lambda_{43}=\sin \gamma_{3}\left[\sin \gamma_{1} \cos \gamma_{2}\left(l_{3}-l_{2}\right)-\sin \gamma_{2} \cos \gamma_{1}\left(\left(l_{f}-l_{r}\right) l_{1} / l-l_{3}\right)\right] \\
& \lambda_{44}=\left(\sin \gamma_{1} \cos \gamma_{2}-\sin \gamma_{2} \cos \gamma_{1}\right) \sin \gamma_{3}\left(l_{f}-l_{4}\right) \\
& \lambda_{45}=\sin \gamma_{1} \cos \gamma_{2} \cos \gamma_{3}\left(l_{3}+\frac{l_{1}\left(l_{f}-l_{4}\right)-l_{2}\left(l_{r}+l_{4}\right)}{l}\right)- \\
& \sin \gamma_{2} \cos \gamma_{1} \cos \gamma_{3}\left(l_{1}+l_{3}\right)+\sin \gamma_{3} \cos \gamma_{1} \cos \gamma_{2} \frac{\left(l_{1}+l_{2}\right)\left(l_{4}-l_{f}\right)}{l} \\
& \lambda_{51}=\sin \gamma_{1} \sin \gamma_{3} \cos \gamma_{2} \cos \gamma_{4} l l_{2}+\sin \gamma_{2} \sin \gamma_{3} \cos \gamma_{1} \cos \gamma_{4}\left(l_{r} l_{1}-l_{f} l_{2}\right)+ \\
& \sin \gamma_{1} \sin \gamma_{4} \cos \gamma_{2} \cos \gamma_{3}\left(l_{f} l_{1}-l_{r} l_{2}\right)+\sin \gamma_{2} \sin \gamma_{4} \cos \gamma_{1} \cos \gamma_{3}\left(l_{r}-l_{f}\right) l_{1} \\
& \lambda_{52}=\left[\begin{array}{l}
\left(\sin \gamma_{2} \cos \gamma_{4}+\sin \gamma_{4} \cos \gamma_{2}\right) \cos \gamma_{1} \cos \gamma_{3}- \\
\left(\sin \gamma_{1} \cos \gamma_{3}+\sin \gamma_{3} \cos \gamma_{1}\right) \cos \gamma_{2} \cos \gamma_{4}
\end{array}\right] l\left(l_{1}+l_{2}\right) \\
& \lambda_{53}=\left(\sin \gamma_{1} \sin \gamma_{4} \cos \gamma_{2} \cos \gamma_{3} l_{2}-\sin \gamma_{2} \sin \gamma_{3} \cos \gamma_{1} \cos \gamma_{4} l_{2}\right)\left(l_{1}+l_{2}\right) \\
& \lambda_{54}=\left[\begin{array}{l}
\sin \gamma_{1} \sin \gamma_{3} \cos \gamma_{2} \cos \gamma_{4}-\sin \gamma_{2} \sin \gamma_{3} \cos \gamma_{1} \cos \gamma_{4}+ \\
\sin \gamma_{2} \sin \gamma_{4} \cos \gamma_{1} \cos \gamma_{3}-\sin \gamma_{1} \sin \gamma_{4} \cos \gamma_{2} \cos \gamma_{3}
\end{array}\right] l \\
& \lambda_{55}=\left[\begin{array}{l}
\sin \gamma_{1} \cos \gamma_{2} \cos \gamma_{3} \cos \gamma_{4}-\sin \gamma_{2} \cos \gamma_{1} \cos \gamma_{3} \cos \gamma_{4}+ \\
\sin \gamma_{4} \cos \gamma_{1} \cos \gamma_{2} \cos \gamma_{3}-\sin \gamma_{3} \cos \gamma_{1} \cos \gamma_{2} \cos \gamma_{4}
\end{array}\right]\left(l_{1}+l_{2}\right)
\end{aligned}
$$

